\title{
A Safe Image Steganography for Verbal Communication through Information Activity in Digital Pictures
}

\author{
Rinesh.S, Nithyapoojeswari.D
}

\begin{abstract}
Steganography is with the intention of the art and science of writing secreted messages in such with the aim of nobody excluding the dispatcher and supposed recipient, suspects the existence of the message. We tend to tend to are constructing Associate in Nursing implementing a fresh algorithm supported activity an oversized amount of information (image, audio, text) file into color BMP image. We have been used adaptive image filtering and adaptive image segmentation with bits replacement on the suitable pixels. These image parts are elite willy-nilly rather than consecutive by using a brand new plan made public by main cases with their sub cases for each store unit in one component. this concept supports every visual and mathematics in line with the steps of fashion, we have been everywhere sixteen main cases with their sub cases that cowl all aspects of the computer file into color icon image. High-security layers are projected through 3 layers to form it arduous to interrupt through the committal to writing of the laptop file and confuse steganalysis too. These techniques are a great deal of useful for investigation the stego footage what is more as a result of the image media regarding security of images and embed the knowledge for advanced image area which we'll merely calculate approximately the high embed rate by victimization the quantitative steganalysis technique.
\end{abstract}

\section{INTRODUCTION}

Digital multimedia structure in order provides a study and easy piece of script and modify of knowledge. The data are frequently delivered over laptop network with very little to no error and sometimes while not intrusion. Sadly, digital media sharing raise a priority for digital substance owners. Digital information could also be derived with none failure in value and content. This pose a massive drawback for the fortification of assets privileges of official document owners. Watermarking may be a answer to the matter. It are often outlined as embed digital information, like info concerning the owner, beneficiary, and access level, while not being detectable within the host multimedia system information. Steganography depends on activity clandestine communication in unsuspected compact disk method information and is mostly employed in secret communication between acknowledged parties.

Revised Manuscript Received on July 13, 2019.

Rinesh.S, Assistant Professor, Department of Computer Science and Engineering, Saveetha School of Engineering, SIMATS, Chennai, Tamilnadu, INDIA.

Nithyapoojeswari.D, UG Scholar, Department of Computer Science and Engineering, Saveetha School of Engineering, SIMATS, Chennai, Tamilnadu, INDIA.

Steganography may be a technique of secret writing that hides information among the bits of a canopy file, like a graphic or Associate in Nursing audio file. The method replace sidle or unimportant bits with the key in sequence. Steganography isn't as strong to attack as the implanted information is liable to destruction.

\section{PROBLEM STATEMENT}

Cryptography has followed through many stage of development. Prehistoric Egyptian inscribe worn substandard hieroglyphics unite in nursing inscription. Hebrew scribes worn ATBASH, an upturned alphabet simple resolution cipher. Cryptography sustained from side to side from the past with more than a few variations. These days cryptography has reach a substitute level. Quantum cryptography combines physics and cryptography to supply a replacement cryptosystem that cannot be crushed whereas not the dispatcher and beneficiary having the knowledge of the tried and unproductive interference. Cryptography defines the art and science of remodeling information keen on a series of bits with the intention of appear as random and unimportant to a surface spectator or aggressor. Cryptanalytic are the reverse engineering of cryptography try to spot weaknesses of assorted cryptanalytic algorithms and their methods to use them. Cryptanalytic is outlined as Associate in nursing attack. Scientific discipline encompasses each cryptography and cryptanalytic and appears at arithmetical issues that lie beneath them. Cryptosystems square measure computer systems accustomed cipher information for secure transmission and storage. Plaintext is message or information that are in their traditional, readable (not crypted) type.

\section{RELATED WORKS}

Chi-Kwong, ChanL.M.Cheng. A knowledge concealing theme through easy LSB replacement is projected. As a result of applying educational measure best picture element adjustment technique to the stego-image obtain via the easy LSB replacement methodology, the picture excellence of the stego-image are going to be deeply enhanced with low further machine complexness. The nastiest case meansquare-error flanked by the stego-image and conjointly the cover-image springs. Results prove that the stego picture is visually impossible to tell apart from the primary cowl picture. The results show a giant improvement with relevancy a previous work. 
PratapChandraMandal.,B.P.Poddar. Steganograpy is one in all the strategies of underground message that hide the subsistence of unseen communication. It'll be printed as a result of the revision of indiscernible message that generally deals with the ways in which of concealing the existence of the communicate note. The concealed note could also be passage, picture, acoustic, videocassette, etc. The files are going to be a cover picture once inserts the note into the cover image victimization stego-key. It's noted as stego-image. Steganography is currently a lot of vital because of the growth and covert communiqué of potential laptop users on the net. I actually have analyzed varied Steganography techniques. Given an outline of Steganography, completely different strategies of Steganography, its applications, however it's completely dissimilar commencing cryptography.

Abbas Cheddad, Joan Condell, Kevin Curran, and Paul Mc Kevitt. Steganography involves message secret in order it associate acceptable transmission carrier, e.g., picture, acoustic, and videocassette files. It comes below the assumption that if the feature is visible, that aspire of assault is apparent, therefore the goal here is usually to cover the really existence of the embedded information. Steganography has varied helpful applications. It is often used for sick intentions. It's been propelled to the forefront of current security techniques by the exceptional growth in procedure power, the rise in security awareness by, e.g., public, group, agency, administration and through intellectual pursuit. Steganography's final objectives, that are undetectability, hardiness (resistance to numerous image process strategies and compression) and capability of the hidden information, are the most factors that separate it from connected techniques like watermarking and cryptography. This document provides a progressive evaluation and examination of the varied existing ways that of Steganography beside some common standards and pointers drawn from the literature. Steganalysis that is that the science of assaultive Steganography isn't the major center of this review however nevertheless are going to be in short mentioned.

Eugene T. Lin and Edward J. Delp. Videocassette and picture process Laboratory (VIPER).We are going to précis the utilization of knowledge activity techniques in digital pictures. In express, we are going to describe however one will use Steganography to cover info in a very digital picture. Steganography is alleged to cryptography and is that the idea for many of the digital watermarking techniques presently being developed. The attention in order activity has up with the recent action in digital exclusive rights guard scheme. A way to protect the possession of a digital picture is to on the letter. T. Plant info at intervals the content of the image distinctive the owner. This document will evaluate recent development in information activity, especially as an effect of elite privileges defender of digital copy.

W. Bender D. Gruhl N. Morimoto A. Lu. Data activity, a sort of Steganography, embed information into digital media for the aim of recognition, footnote, and exclusive rights many constraint have an impact on this process: the number of knowledge to be concealed, the requirement for changelessness of those information underneath conditions wherever a "host" sign is focus to distortions, e.g., lossy density, and therefore the amount to that in order should be resistant to interception, alteration, or taking away by a 3rd party. We tend to explore every ancient and novel technique for address the data-hiding method and price these techniques in light-weight of three applications: exclusive rights shield, interfere proofing, and rise in order embedding.

\section{EXISTING SYSTEM}

Cryptography has various stages of progression. Earliest Egyptian engraves used substandard hieroglyphics in Associate in nursing inscription. Hebrew scribes used ATBASH, an upturned alphabet easy reply cipher. Cryptography continued through history with several variations. Now a day's cryptography has reached a newest level. Quantum cryptography combines physics and cryptography to give a latest cryptosystem that cannot be defeated whereas not the dispatcher and beneficiary having the information of the tried and failing intrusion.

\section{Dis-Advantages}

Cryptography scramble a note therefore it cannot be unstated. In Cryptography, a cipher message, for example, would possibly stimulate doubt on the part of the beneficiary whereas Associate in nursing invisible message created with Steganography strategies won't. It takes a protracted time to work out the code. If you wish to send a code to a different person within the past, it'll take very long time to induce thereto person. Overall cryptography can be a protracted technique. Transmission time for documents encrypted victimization public key cryptography is considerably slower. Transmission of terribly massive documents is prohibitory. The input sizes should be significantly better to attain the elevated level of shield.

\section{PROJECTED SYSTEM}

\section{Advantages of projected system}

The plain benefit of Steganography more than cryptography is that communication doesn't draw any consideration. An oblique note that's unhidden, despite however strong the secret writing, can stimulate doubt and should in itself be tricky. In some countries secret script is unlawful. Stego could even be varied with clandestine script therefore the carrier file really carries a note that's encrypted. Therefore though intercept, one more barrier is conferred in making an attempt to interrupt the secret writing.

\section{Objective}

The target of this project is to supply Associate in Nursing economical information activity technique and secret writing within which the image and therefore the audio are often retrieved severally.

\section{Motivation}

To insert over single small piece at every store unit in one 
constituent of the cover-picture and procure the outcome a bit like the LSB. This could be reached by mounting a Steganography algorithmic program to cover great sum of a few sort of info through JPG picture by victimization most range of bits per computer memory unit at every picture element. Any sort of information is often concealed in a very JPG picture that have 24- bit by victimization the Steganography algorithmic program. The twenty four bits has 3 bytes of RGB colors, every computer memory unit has four bits referred to as Nibbles. The very best worth is hold on within the left nibble and therefore the lowly worth is restricted within the correct gnaw in a very computer memory unit $[1,2]$.

\section{Scope}

Action the knowledge at intervals a picture is commonest process worn at the current. From side to side the web covert messages are often unfold simply by insert the key note in a picture. To hide a communication within a picture while not sterilization its able to be seen property, "noisy" areas that contain color change are often modified intended forduvet picture. The universal method wont to build the change occupied is that the practice of the smallest amount important Bit (LSB), mask, filter and transformation on the duvet picture.

\section{Proposed Algorithmic program}

Steganography is that the art of activity the survival of knowledge in one more show standard to achieve secret communication. It provides invisible communication .In the gift Steganography algorithmic program, two-part (data activity at the dispatcher aspect and at extracting the beneficiary side).RC5 algorithm is used in this method. Comparisons are done with Rc5, Blowfish and DES AES algorithms.

\section{MODULE DESCRIPTION}

1) Sending section Picture segmentation is that the technique of partition a digital picture into numerous segments (sets of pixels, conjointly called super-pixels). The objective of segmentation is to alter and/or change the design of a picture into single subject that is more essential and easier to study. Picture segmentation is typically wont to discover substance and restrictions (lines, curves, etc.) in pictures. A lot of exactly, picture segmentation is that the way of allotment a label to each picture element in a picture such pixels with a comparable tag split sure characteristics. The consequences of picture segmentation might also be a collection of segments that collectively cowl the entire picture or a bunch of contours extracted from the picture (see edge detection). Each of the pixels all through a region area like with connation some feature or compute possessions, like color, intensity, or texture. Adjoining region are significantly in total fully completely dissimilar with association steady feature(s). As soon as functional to a heap of pictures, typical in medical imaging, the resulting contour when picture segmentation are frequently wont to revolve out $3 \mathrm{D}$ reform with the help of exclamation algorithms similar to march cube.

2) Cover image A picture that hold secret message.
3) Embedding provided many text embedding modules in not solely English however conjointly in many different languages like German, Korean, Japanese, etc. (At the time of scripting this article), several of them turn out embeddings at the sentence level and not at the word level.

4) Audio activity Image augmentation is a vital part within the coaching pipeline to extend the accuracy of the model. None of the image augmentation modules (at the time of writing) have any variables in them, and consequently, these modules do not appear to be fine-tunable/trainable.

5) Showing steganography image Object detection modules don't support fine-tuning, therefore you may need to perform coaching from scratch if you've got your own dataset. Batching of the information is additionally not supported at the instant.

6) Generator Modules these correspond to Generative Adversial Networks (GAN). A number of the modules haven't exposed their soul a district of the network.

\section{Receiving section}

Steganography image extraction. Audio output.

\section{SYSTEM ARCHITECTURE}

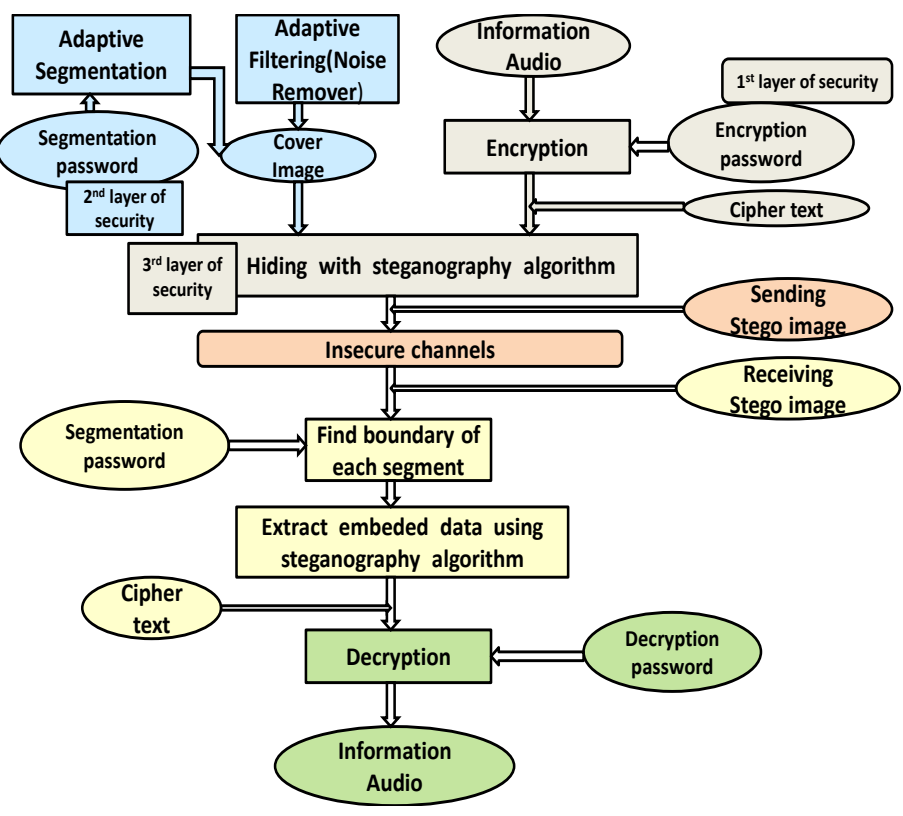

Fig. 1 System Architecture

\section{STEGANOGRAPHY ENCRYPTION}

It is really the study of concealing data from individuals who might snoop on you. The contrast among Steganography and encryption is that the future snoopers will most likely be unable to tell there's any concealed data in any case. 


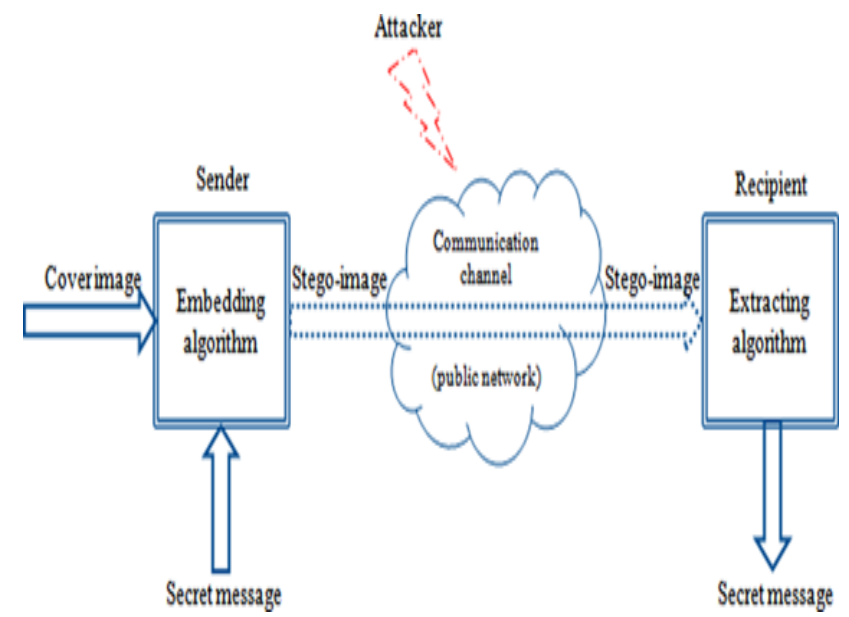

Fig. 2 Steganography Encryption

This is the most straightforward sort of encryption that includes just a single mystery key to figure and translate data. Even encryption is an old and best-known system. It utilizes a mystery key that can either be a number, a word or a string of arbitrary letters. It is a mixed with the plain content of a message to change the substance with a certain goal in mind. The sender and the beneficiary should realize the mystery key that is utilized to encode and unscramble every one of the messages. Blowfish, AES, DES, and RC5 are instances of symmetric encryption. The below diagram shows the Embedded Input and Audio Output.

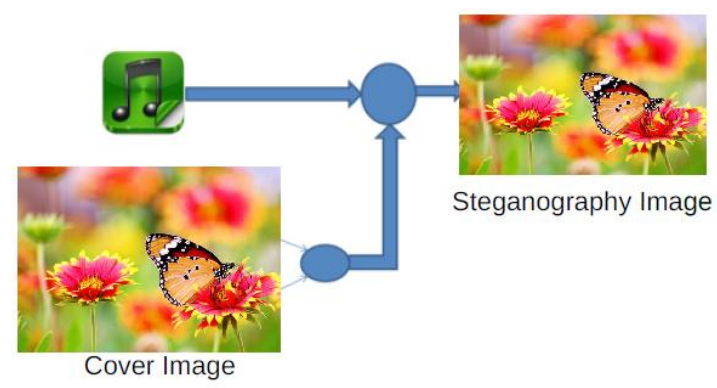

Fig. 3 Embedded Inputs

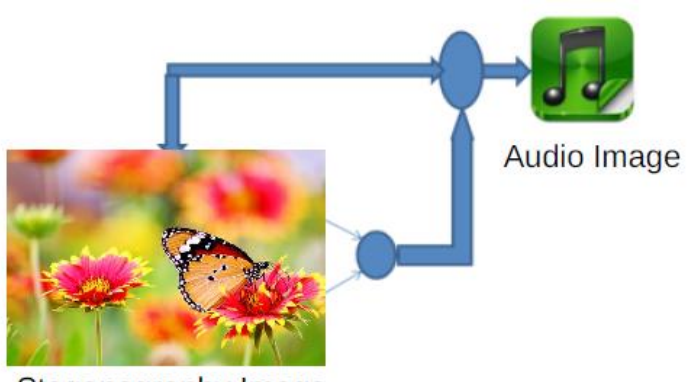

Steganography Image

Fig. 4 Audio Output

\section{RESULT ANALYSIS}

RC5 algorithm performs more rapidly than AES, DES\& Blowfish. Fig. 5 shows the average execution time for these four algorithms to execute the files. According to fig.5 RC5 is faster than AES, Blowfish and DES. Performance comparisons are shown in below Table 1 .
Table. 1 Performance comparison on the basis of execution time

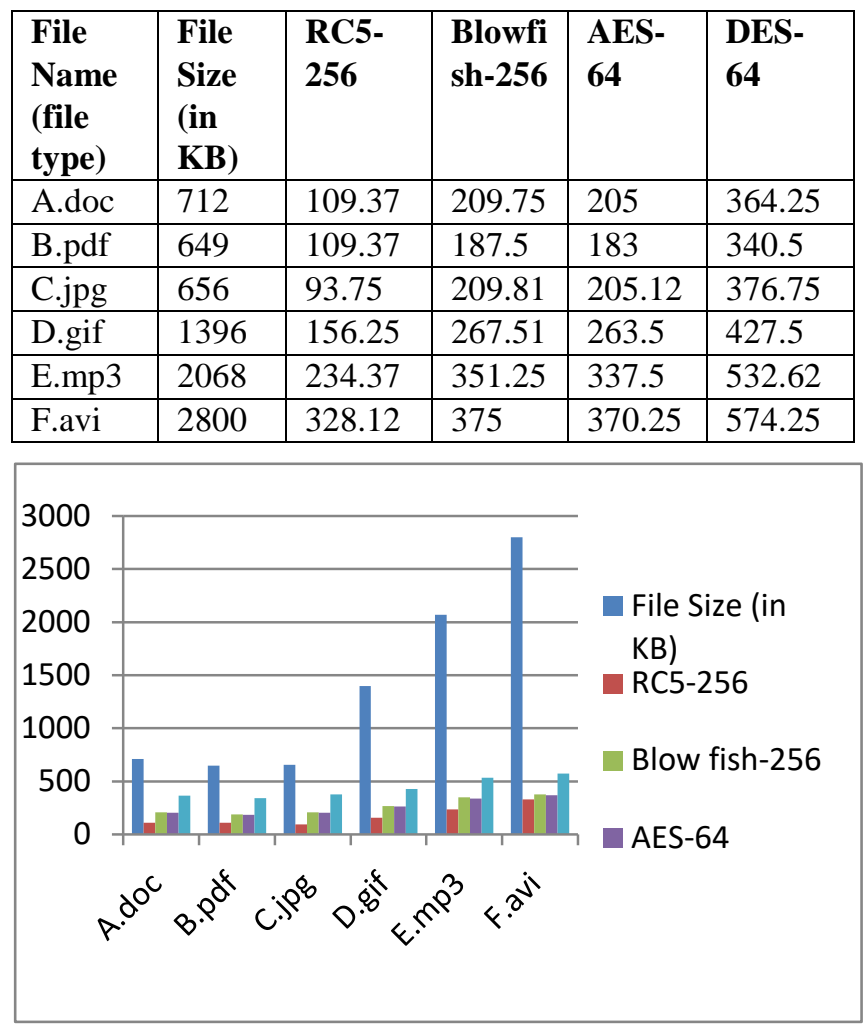

Fig. 5 Average Execution Time of Algorithms

Based on the above results Signal to noise ratio have improved from 57.2 to 98.3.Security have been increased due to RC5 algorithm.

\section{FUTURE ENHANCEMENT}

The projected technique modifies the duvet picture file to imbed key note. To extend the protection of the planned theme, we tend to use an input to regulate the activity technique. The experiment shows that our method is safe, inaudible and might be used for activity in order within the picture file. Within the prospect analysis, we tend to arrange to use the mistake alteration system to make bigger the hardiness of this theme. At the end, practicability of picture Steganography was evaluated by considering it's the professionals and cons. In summary, if enforced properly with cryptanalytic strategies to safe the embedded information before placing to a canopy intermediate, several information activity strategies delineate higher than could become powerful tools for the transmission of undetectable and secure communication.

\section{CONCLUSION}

The projected method modifies the amplitude of the duvet picture file to imbed the key note. To extend the safety of the projected theme, we've got a bent to use a key to manage the concealment technique. The experiment shows that our technique is secure, unbearable and should be used for concealment info at intervals the image file. 
Within the future analysis, we tend to arrange to use the fault alteration system to extend the hardiness of this theme. The practicability of picture Steganography be evaluated by considering it's the professionals and cons. In summary, if enforced properly with cryptanalytic strategies to secure the embedded information previous to insertion to a canopy standard, several of the information activity strategies delineate higher than might become powerful tools for the transmission of undetectable and secure communication.

\section{REFERENCE}

1. R.Anderson and F. Petitcolas, "On the boundaries of Steganography" IEEE Journal of elite Areas in Communications, Vol. 16, No. 4, May 1998.

2. Niels Provos, Peter Honeyman, "Hide and Seek: Associate in Nursing Introduction to Steganography," IEEE laptop society, 2003.

3. K B Raja, Venugopal K R and L M Patnaik, "A Secure Steganography algorithmic program victimization LSB, DCT and compression on Raw Images", Technical Report, Department of applied science and Engineering, University Visvesvaraya faculty of Engineering, Bangalore University, December 2004.

4. An outline of image Steganography by T. Morkel, J.H.P. Eloff, M.S. Olivier. info and laptop Security design (ICSA) analysis cluster Department of applied science University of Pretoria national capital $\}$, 0002, Pretoria, South Africa.

5. Johnson, N.F. Jajodia, S.,'Exploring Steganography: Seeing the Unseen", laptop Journal, February 1998.

6. "Detecting LSB Steganography in Color and Gray-Scale Images" Jessica Fridrich, Miroslav Goljan, and Rui Du State University of latest dynasty, Binghamton.

7. Ran-Zan Wang, Chi-Fang Maya Lin, Ja-Chen Maya Lin, "Hiding information in pictures by optimum moderately significant-bit replacement" IEE lepton. Lett. 36(25) (2000) 20692070.

8. Aliza Sarla, Emmanuel O.C Mkpojiogu, "Cloud Information Security using and Steganography Paired Encryption”, International Innovative Research Journal of Engineering and Technology, 4(3), 2019. 